\title{
Maintenance of Antimicrobial Susceptibility of Acinetobacter baumannii in Modeled Microgravity
}

\author{
DONNA M HENSLEY
}

OBJECTIVE: The purpose of this study was to evaluate the effect of a modeled microgravity environment on the antimicrobial resistance of Acinetobacter baumannii.

DESIGN: Clinical isolates of $A$. baumannii $(\mathrm{n}=10)$ in Mueller Hinton broth were grown in high aspect ratio vessels using a rotary cell culture system oriented to achieve either normal earth gravity (1XG) or low sheer modeled microgravity (LSMMG) conditions. After culture MICs were determined by Etest. Antimicrobials included in the study were amikacin, ampicillin/ sulbactam, azithromycin, ceftazidime, gatifloxacin, gentamicin, imipenem, meropenem, minocycline, ticarcillin/clavulanic acid, tigecycline, and trimethoprim/sulfamethoxazole. Results were compared for MIC value and category interpretation (S, I, R).

RESULTS: Overall category agreement was $100 \%$ (360/360). 100\% of MIC values compared were within one twofold dilution of each other.

CONCLUSION: Under these test conditions LSMMG had no apparent effect on the resistance pattern of $A$. baumannii.

ABBREVIATIONS: $1 \mathrm{XG}=$ normal earth gravity; LSMMG = low-shear modeled microgravity, MIC = minimum inhibitory concentration; $S=$ susceptible; $\mathrm{I}=$ intermediate; $\mathrm{R}$ = resistant; NASA = National Aeronautics and Space Administration; RWV = rotating wall vessel; MDR = multi-drug resistant; TSA II - trypticase soy agar with $5 \%$ sheep blood; WHMC $=$ Wilford Hall Medical Center; $\mathrm{MHB}=$ cation adjusted Mueller Hinton Broth; HARV = high aspect ratio vessel; RCCS = rotary cell culture system; $\mathrm{mL}=$ milliliter; $\mathrm{RPM}=$ rotations per minute.
INDEX TERMS: Acinetobacter baumannii, low-shear modeled microgravity, antimicrobial resistance

Clin Lab Sci 2010;23(2):84

Donna Hensley MT(ASCP), Lackland AFB, TX 782369908

Address for Correspondence: Donna Hensley MT(ASCP),

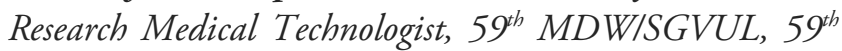
Clinical Research Division, 2200 Bergquist Dr, Bldg 4430, Lackland AFB, TX 78236-9908. (210) 292-6690, (210) 292-2897 (fax), donna.hensley@lackland.af.mil.

Acknowledgements: This research was performed under the authority of the Department of Defense and the $59^{\text {th }}$ Medical Wing, 59 $9^{\text {th }}$ Clinical Research Division, Lackland AFB, TX, Institutional Review Board.

\section{INTRODUCTION}

Growth and development of life on earth are affected by gravity. ${ }^{1-2}$ The advent of space flight introduced questions regarding the effect of zero gravity, or weightlessness, on the human body. Scientists were faced with a myriad of possibilities to consider, one of which was the interaction of man and bacteria under zero or decreased gravity conditions. Although the diversity of microorganisms that have been investigated during spaceflight in the past 30 years is extensive the opportunity to conduct microbiological research in space is restricted by many factors such as demands on the astronauts' time and the difficulties of containment in an enclosed environment. ${ }^{3-5}$ To meet the requirements for microbiological research in a microgravity environment NASA developed a rotating wall vessel (RWV) that created a low-shear force environment for culture of tissue cells and bacteria that mimicked the environmental conditions encountered in earth orbit. The environment created in the RWV has been termed 


\section{RESEARCH AND REPORTS}

Low-Shear Modeled Microgravity (LSMMG) and not only mimics the space flight environment but also lowshear environments in the human body such as those found in utero and between the brush border microvilli of epithelial cells. ${ }^{3,6-8}$

Reports in the literature have shown that bacterial phenotypic characteristics may differ in cells grown in LSMMG when compared to control cultures grown in normal earth gravity (1XG) but that the phenotypic changes in the characteristics can not be generally applied to all bacteria. For example, the growth rate of one bacterium may be increased in LSMMG while another is decreased. Other changes noted in bacteria studied are increased virulence, changes in stress response, changes in antibiotic susceptibility profiles, and increased biofilm formation. ${ }^{3,6,7,9-17}$ Recently publications of molecular studies report up and down regulation of genes in bacteria grown in LSMMG when compared to $1 \mathrm{XG}$ cultures. 3, 7, 11, 15, 18-21

Acinetobacter baumannii is a Gram negative bacterium commonly found in nature and in the hospital environment. It is able to survive on both moist and dry surfaces, human skin and in food. It is also characterized by a high level of antimicrobial resistance. These characteristics have lead to an increasing concern for $A$. baumannii as a potential agent for hospital acquired infections.

Reports in the literature cite an increase in $A$. baumannii infections among patients at military medical facilities treating U.S. service members who have been injured in Iraq and Afghanistan. ${ }^{22,}{ }^{23}$ Other studies, involving environmental and colonization cultures, indicate that the source of the A. baumannii infections is nosocomial in origin. ${ }^{24-26}$ A study at Brook Army Medical Center, Fort Sam Houston, TX, determined the antimicrobial susceptibilities of $142 \mathrm{~A}$. baumannii-calcoaceticus complex isolates, 95 of which were from wounded U.S. soldiers deployed overseas, and found broad antimicrobial resistance among the isolates tested. The isolates from deployed patients were more resistant than the isolates from nondeployed patients though the reason for this is unclear. ${ }^{27} \mathrm{An}$ independent association between patients with multidrug resistant (MDR) Acinetobacter infection and increased hospital and intensive care unit length of stay has been found when compared to patients with susceptible Acinetobacter infection. ${ }^{28}$ The appearance of MDR A. baumannii in both military and civilian medical facilities represents a growing threat to the healthcare community. ${ }^{29-32}$ Research into the growth and resistance mechanisms of this organism is needed to facilitate the development of new treatment methods.

The purpose of this study was to evaluate the effect of a low-shear force environment on the antimicrobial resistance of Acinetobacter baumannii. Investigation into the genomic or proteomic mechanisms of any discovered resistance pattern changes could provide new treatment targets for this emerging pathogen.

\section{MATERIALS AND METHODS}

Clinical isolates of $A$. baumannii $(\mathrm{n}=10)$ were isolated from cultures submitted as standard of care to the microbiology laboratory at Wilford Hall Medical Center (WHMC), Lackland AFB, TX, and were frozen at $-70^{\circ} \mathrm{C}$ until testing. Isolates were transferred to the WHMC Clinical Research Division microbiology laboratory for testing. Before testing each isolate was subcultured twice to trypticase soy agar with 5\% sheep blood (TSA II) (BBL/BD, Sparks, MD). On test days a suspension of the $A$. baumannii isolate was prepared by harvesting cells from $16-24$ hour growth on the TSA II plate and suspending the cells in cation adjusted Mueller Hinton Broth (MHB) (BBL/BD, Sparks, MD) and adjusting the turbidity to equal a $0.5 \mathrm{McF}$ arland turbidity standard (approximately $1.5 \times 10^{8}$ cells $/ \mathrm{mL}$ ). This suspension was then diluted 1:200 in MHB to achieve a working suspension of approximately $7.5 \mathrm{x}$ $10^{5}$ cells $/ \mathrm{mL}$. $10 \mathrm{~mL}$ high aspect ratio vessels (HARVs) were conditioned by filling them with sterile MHB for at least 10 minutes. The conditioning $\mathrm{MHB}$ was discarded and the HARVs were filled with the working suspension of $A$. baumannii. Care was taken to completely fill the HARVs and to remove all air bubbles. HARVs were placed on a rotary cell culture system (RCCS) (Synthecon, Inc., Houston, TX) oriented to achieve either 1XG or LSMMG conditions (Figures 1 and 2) and incubated for $4-5$ hours at $37^{\circ} \mathrm{C}$, ambient air. Rotation of the HARVs was counterclockwise at 25 RPM. After incubation the culture broth was removed from the HARVs, adjusted to 0.5 


\section{RESEARCH AND REPORTS}

McFarland turbidity and used for MIC testing. MICs were determined by Etest (AB Biodisk North America, Piscataway, NJ). Briefly, the culture broth was applied to the surface of a Mueller Hinton Agar plate with a cotton-tipped swab. The plates were allowed to stand for 10-15 minutes and then the Etest strips were applied to the agar surface. Within 1 hour of strip application the plates were incubated for $16-24$ hours at $34-35^{\circ} \mathrm{C}$ in ambient air. After incubation MICs were determined by removing the cover of the Petri dish, holding the plate to transmitted light, and reading the MIC at the point where growth intersected the Etest strip. Tests were set up in triplicate. Antimicrobials included in the study were amikacin, ampicillin/sulbactam, azithromycin, ceftazidime, gatifloxacin, gentamicin, imipenem, meropenem, minocycline, ticarcillin/clavulanic acid, tigecycline, and trimethoprim/sulfamethoxazole. Results were compared for MIC value and category interpretation of susceptible, intermediate, or resistant (S, I, R).

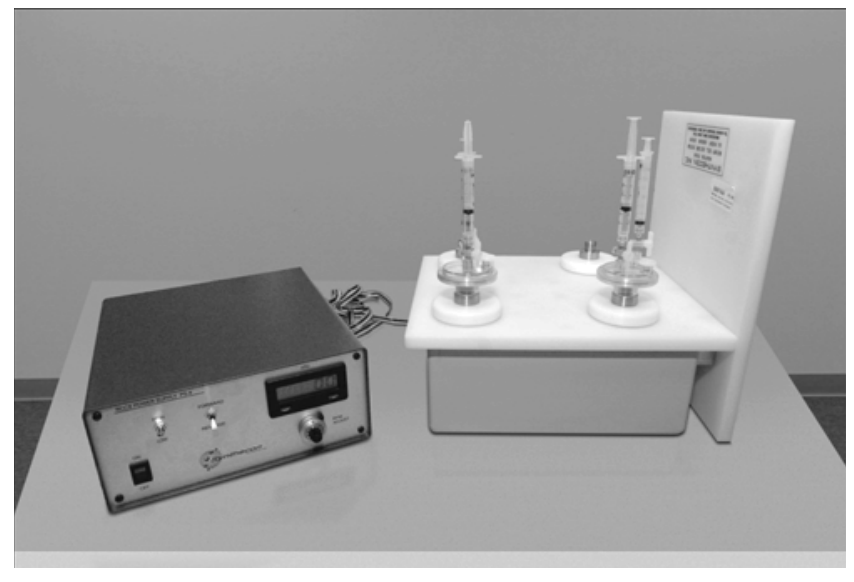

Figure 1. Rotary Cell Culture System (RCCS) in normal earth gravity (1XG) position.

\section{RESULTS}

There was a variety of resistance patterns seen with the ten $A$. baumannii isolates. Table 1 . Under these test conditions there was no difference seen in the resistance patterns of the isolates when grown under LSMMG and 1XG. Overall category agreement was 100\% (360/360) and $100 \%$ of MIC values compared were within one twofold dilution of each other.

\section{DISCUSSION}

The ever increasing impact of MDR organisms on the healthcare environment is a cause of concern for all healthcare professionals. Insight into the mechanisms of drug resistance is important if successful treatment of MDR organisms is to be achieved and there are many avenues of investigation open for study in this area. This study looked for effects on the resistance pattern of a small group of $A$. baumannii clinical isolates under a very specific set of conditions. Although no changes in resistance patterns were detected the study gives rise to a variety of other possibilities for future research.

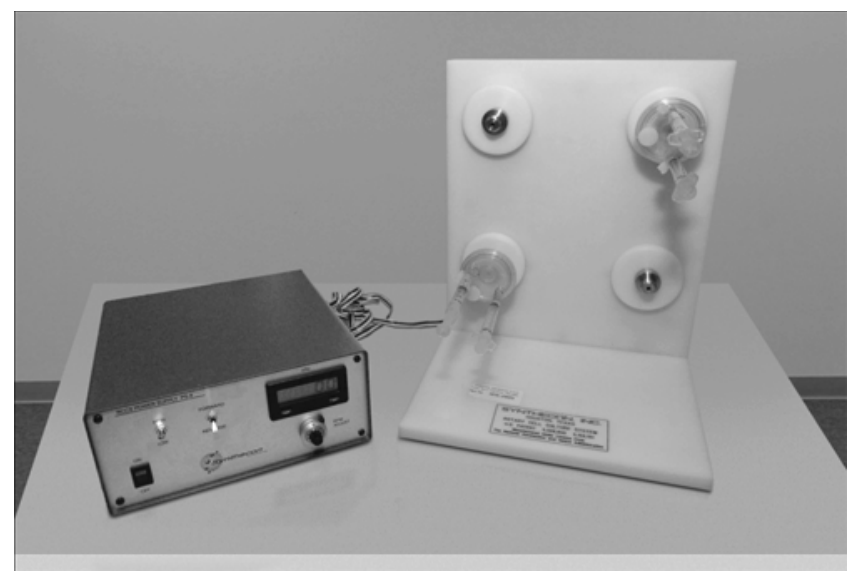

Figure 2. Rotary Cell Culture System (RCCS) in low-shear modeled microgravity (LSMMG) position.

Only one speed of rotation was evaluated in this study. 25 RPM was selected because it is commonly cited in the literature as the speed of rotation used by other researchers. Some of the published work uncovered marked changes in microorganisms grown in HARVs under $1 \mathrm{XG}$ and LSMMG at $25 \mathrm{RPM}$. It is possible that this is not the optimal speed of rotation needed to achieve maximum low-shear force for $A$. baumannii. Other speeds of rotation could be evaluated.

The length of time the isolates were exposed to LSMMG was limited to 4-5 hours in this study. It is possible that 4-5 hours is not sufficient for the effects of incubation in LSMMG to become detectable. In an effort to determine if this was the case one isolate was incubated under LSMMG conditions for 7 days in M9 Minimal Broth before being transferred to MHB and incubated for Etest. To prevent overcrowding in the HARV over the seven day period a fresh 1:200 dilution 
Table 1. Susceptibility patterns for individual A. baumannii isolates. Results are from triplicate testing at both 1XG and LSMMG.

\section{ISOLATE NUMBER}

\begin{tabular}{|c|c|c|c|c|c|c|c|c|c|c|}
\hline Antimicrobial Name & 1 & 2 & 3 & 4 & 5 & 6 & 7 & 8 & 9 & 10 \\
\hline Amikacin & S & $\mathrm{I}$ & $\mathrm{I}$ & $\mathrm{R}$ & S & S & S & S & S & S \\
\hline Ampicillin/sulbactam & $\mathrm{R}$ & S & S & $\mathrm{R}$ & S & S & S & S & S & S \\
\hline Azithromycin & $\mathrm{R}$ & $\mathrm{R}$ & $\mathrm{R}$ & $\mathrm{R}$ & $\mathrm{R}$ & $\mathrm{R}$ & $\mathrm{R}$ & $\mathrm{R}$ & $\mathrm{R}$ & $\mathrm{R}$ \\
\hline Ceftazidime & $\mathrm{R}$ & $\mathrm{R}$ & $\mathrm{I}$ & $\mathrm{R}$ & $\mathrm{R}$ & $\mathrm{R}$ & S & $\mathrm{S}$ & S & S \\
\hline Gatifloxacin & $\mathrm{R}$ & $\mathrm{R}$ & $\mathrm{I}$ & $\mathrm{R}$ & $\mathrm{R}$ & $\mathrm{R}$ & S & S & S & S \\
\hline Gentamicin & $\mathrm{R}$ & $\mathrm{R}$ & $\mathrm{R}$ & $\mathrm{R}$ & S & S & S & S & S & S \\
\hline Imipenem & $\mathrm{R}$ & $\mathrm{R}$ & $\mathrm{R}$ & $\mathrm{R}$ & S & S & S & S & S & S \\
\hline Meropenem & $\mathrm{R}$ & $\mathrm{R}$ & $\mathrm{R}$ & $\mathrm{R}$ & S & S & S & S & S & S \\
\hline Minocycline & S & $\mathrm{S}$ & $\mathrm{S}$ & $\mathrm{R}$ & S & S & S & S & S & S \\
\hline Ticarcillin/clavulanic acid & $\mathrm{R}$ & $\mathrm{R}$ & $\mathrm{R}$ & $\mathrm{R}$ & $\mathrm{R}$ & $\mathrm{R}$ & S & S & S & S \\
\hline Tigecycline & $\mathrm{R}$ & I & $\mathrm{R}$ & $\mathrm{R}$ & $\mathrm{I}$ & $\mathrm{I}$ & S & S & S & S \\
\hline Trimethoprim/sulfamethoxazole & $\mathrm{R}$ & $\mathrm{R}$ & $\mathrm{R}$ & $\mathrm{R}$ & $\mathrm{R}$ & $\mathrm{R}$ & S & S & $\mathrm{S}$ & S \\
\hline
\end{tabular}

was made of the culture daily. The dilution was made by drawing off old culture broth and replacing it with fresh M9 broth without removing the HARV from rotation on the rotator base. The exchange was done slowly to prevent the creation of turbulence within the HARV. There was no difference in the Etest set up on the isolate after one week exposure to LSMMG when compared to the 1XG Etest. It is also possible that the 4-5 hour incubation in the HARVs was too long. Most of the culture broths removed from the HARVs after 45 hours had achieved turbidity greater than a 0.5 McFarland standard and had to be adjusted down to the correct turbidity before Etests could be set up. Perhaps overcrowding in the HARVs prevented the maintenance of a true LSMMG environment. Other lengths of time of exposure to LSMMG could be investigated.

One other possibility to consider is that changes did occur during incubation under LSMMG but that the changes were easily and rapidly reversed when incubated under the 1XG environment used for the Etest. It might be possible to set up an MIC in the HARVs so that the entire MIC could be performed under LSMMG conditions.

While a change in the phenotypic resistance pattern of A. baumannii was not detected by the test conditions utilized in this study it is possible that the optimum conditions were not achieved to create and maintain the
LSMMG environment. It is also possible that under these test conditions changes not detectable by Etest, such as proteomic, may have occurred. Further studies are needed to fully evaluate the effects of LSMMG on the resistance mechanisms of $A$. baumannii.

Disclaimer: This material represents the personal statements of the authors and is not intended to constitute an endorsement by the $59^{\text {th }}$ Medical Wing or any other federal entity.

\section{REFERENCES}

1. Horn ER. Gravity effects on life processes in aquatic animals. [Review] [148 refs] Adv Space Biol Med. 2005;10:247-301.

2. The effects of gravity on plant growth and development. Available from http://today.uconn.edu/?p=3302. Accessed 2009 Sept 16.

3. Nickerson CA, Ott CM, Wilson JW, Tamamurthy R, and Pierson DL. Microbial responses to microgravity and other low-shear environments. Micro and Mol Biol Rev 2004;68(2):345-61.

4. Mishra SK and Pierson DL. Space flight: effects on microorganisms, p. 53-60. In J. Lederberg (ed.), Encyclopedia of microbiology, vol. 4. Academic Press, Inc., San Diego, CA. 1992.

5. Klaus DM. Space microbiology: microgravity and microorganisms, p. 2996-3004. In G. Bitton (ed.), Encyclopedia of environmental microbiology. John Wiley \& Sons, Inc., New York, N.Y. 2002.

6. "Modeled microgravity" increases virulence of common bacterial pathogen. Space Life Sciences Research Highlights. Office of Biological and Physical Research, National Aeronautics and Space Administration, 2001. 


\section{RESEARCH AND REPORTS}

7 Nickerson CA, Ott CM, Wilson JW, Ramamurthy R, et al. Low-shear modeled microgravity: a global environmental regulatory signal affecting bacterial gene expression, physiology, and pathogenesis. J of Micro Methods 2003;54:111.

8 Nauman EA, Ott CM, Sander E, Tucker DL, et al. Novel quantitative biosystem for modeling physiological fluid shear stress on cells. Appl and Environ Micro 2007;73(3):699-705.

9 Nickerson CA, Goodwin TJ, Terlonge J, Ott CM, et al. Three-dimensional tissue assemblies: novel models for the study of Salmonella enterica serovar Typhimurium pathogenesis. Inf and Imm 2001;69(11):7106-20.

10 Demain AL and Fang A. Secondary metabolism in simulated microgravity. Chem Rec 2001;1(4):333-46.

11 Tucker DL, Ott CM, Huff S, Fofanow Y, et al. Characterization of Escherichia coli MG 1655 grown in a lowshear modeled microgravity environment. BMC Micro 2007; 7:15-30.

12 Carterson AJ, Honer zu Bentrup K, Ott CM, et al. A549 lung epithelial cells grown as three-dimensional aggregates: alternative tissue culture model for Pseudomonas aeruginosa pathogenesis. Inf and Imm 2005;73(2):1129-40.

13 Chopra V, Fadl AA, Sha J, Chopra S, et al. Alterations in the virulence potential of enteric pathogens and bacterial-host cell interactions under simulated microgravity conditions. J Tox and Env Health, Part A 2006;69:1345-70.

14 Baker PW and Leff L. The effect of simulated microgravity on bacteria from the Mir space station. Microgravity Sci Technol 2004;15(1):35-41.

15 Nickerson CA, Ott CM, Mister SJ, Morrow BJ, et al. Microgravity as a novel environmental signal affecting Salmonella enterica serovar Typhimurium virulence. Inf and Imm 2000;68(6):3147-52.

16 Lynch SV, Mukundakrishnan K, Benoit MR, Ayyaswamy PS, and Matin A. Escherichia coli biofilms formed under low-shear modeled microgravity in a ground-based system. Appl and Environ Microbiol 2006;72(12):7701-10.

17 Tucker DL, Ott CM, Huff S, Fofanow Y, et al. Characterization of Escherichia coli MG 1655 grown in a lowshear modeled microgravity environment. BMC Microbiology 2007;7:15.

18 Lynch SV and Matin A. The stress response of Escherichia coli to conditions of simulated microgravity. Abstract I-35: Amer Soc for Micro $103^{\text {rd }}$ General Meeting 2003.

19 Wilson JW, Ramamurthy R, Porwollik S, McClelland M, et al. Microarray analysis identifies Salmonella genes belonging to the low-shear modeled microgravity regulon. PNAS 2002;99(21):13807-812.
20 Wilson JW, Ott CM, Ramamurthy R, Porwollik S, et al. Lowshear modeled microgravity alters the Salmonella enterica serovar Typhimurium stress response in an RpoS-independent manner. Appl and Environ Micro 2002;68(11):5408-16.

21 Nauman EA, Ott CM, Sander E, Tucker DL, et al. Novel quantitative biosystem for modeling physiological fluid shear stress on cells. Appl and Environ Micro 2007;73(3):699-705.

22 Centers for Disease Control and Preventions. Acinetobacter baumanii infections among patients at military medical facilities treating injured US service members, $2002-2004$. MMWR Morb Mortal Wkly Rep 2004;53:1063-6.

23 Davis KA, Moran KA, McAllister CK, and Gray PJ. Multidrug resistant Acinetobacter extremity infections in soldiers. Emerg Infec Dis 2005;11(8):1218-24.

24 Griffith ME, Ellis MW, and Murray CK. Acinetobacter nares colonization of healthy US soldiers. Inf Cont and Hosp Epidem 2006;27(7):787-8.

25 Murray CK, Roop SA, Hospenthal DR, Dooley DP, et al. Bacteriology of War Wounds at the Time of Injury. Military Med 2006;171(9):826-9.

26 Scott P, Deye G, Srinivasan A, Murray C, et al. An outbreak of multidrug-resistant Acinetobacter baumanii-calcoaceticus complex infection in the US military health care system associated with military operations in Iraq. Clin Inf Dis 2007;44(12):1577-84.

27 Hawley JS, Murray CK, Griffith ME, McElmeel ML, et al. Susceptibility of Acinetobacter strains isolated from deployed U.S. military personnel. Antimicrob Agents and Chemo 2007;51(1):376-8.

28 Sunenshine RH, Wright MO, Maragakis LL, Harris AD, et al. Multidrug-resistant Acinetobacter infection mortality rate and length of hospitalization. Emer Inf Dis 2007;13(1):97-103.

29 Lolans K, Rice TW, Munoz-Price LS, and Quinn JP. Mulitcity outbreak of carbapenem-resistant Acinetobacter baumannii isolates producing the carbapenemase OXA-40. Antimicrob Agents and Chemother 2006;50(9):2941-5.

30 Maslow JN, Glaze T, Adams P, Lataillade M. Concurrent outbreak of multidrug-resistant and susceptible subclones of Acinetobacter baumannii affecting different wards of a single hospital. Infect Control Hosp Epidemiol 2005;26(1):69-75.

31 Quale J, Bratu S, Landman D, Heddurshetti R. Molecular epidemiology and mechanisms of carbapenem resistance in Acinetobacter baumannii endemic in New York City. Clin Infect Dis 2003;37(2):214-20.

32 Adams-Haduch JM, Paterson DL, Sidjabat HE, et al. Genetic basis of multidrug resistance in Acinetobacter baumannii clinical isolates at a tertiary medical center in Pennsylvania. Antmicrob Agents Chemother 2008;52(11):3837-43. 